\title{
The Study of Barred Spiral Galaxies with Integral-Field Units
}

\author{
Carmelle Robert ${ }^{1}$, Simon Cantin ${ }^{1}$, Élaine Brière ${ }^{1,2}$, \\ Laurie Rousseau-Nepton ${ }^{1}$, and Laurent Drissen ${ }^{1}$ \\ ${ }^{1}$ Université Laval \& CRAQ, Québec, QC, G1V 0A6, Canada, \\ email: carobert@phy.ulaval.ca \\ ${ }^{2}$ Royal Military College, Kingston, ON, K7K 7B4, Canada
}

\begin{abstract}
Integral-Field Units have been used to describe the morphology in numerous continuum and line wavebands of a sample of barred spirals galaxies (some of which known as starbursts and LINERs) and to characterize their gas and stellar populations. The central region of eight galaxies was studied with OASIS and an iterative technique developed to separate superposed stellar populations. Young populations, responsible for nebular emission lines, are found in nuclear structures. Older stellar components are mostly uniformly distributed. The mass distribution of the different populations indicates a long sequence of burst events over the whole galaxy history. An activity of the type composite/transition region is often seen over the whole central region. SpIOMM was also used to map the emission lines over the entire disk of one of these galaxies. Among other results, it confirms the peculiar line ratio.
\end{abstract}

Keywords. Galaxies: spiral, galaxies: stellar content, galaxies: bulges, galaxies: evolution

\section{Galaxy Evolution}

The assembly of the components of a galaxy is a puzzling but important subject when describing galaxy evolution. Among various key elements, interactions, mergers, or cosmic filaments are considered to play a role in the accretion of gas and its flow process (Combes 2008). Large scale bars are also believed to be an efficient way to drive gas toward the central kiloparsecs of the galaxy (Norman et al. 1996). A nuclear structure (bar, ring...) is often observed than can also drive the gas deeper in the central region and trigger nuclear starbursts or turn on a central engine (Regan \& Teuben 2004; Haan et al. 2009). For late-type spirals, internal or environmental processes have been proposed as a mechanism for the formation of a pseudobulge (Kormendy \& Kennicutt 2004).

We are approaching the problematic of galaxy formation and evolution by a detailed study of the stellar generations that are sculpting a galaxy over time. By describing the characteristics (age, metallicity, mass...) of the various stellar populations seen with high spatial resolution, we hope to be able to rebuild the history of galaxies and find clues for identifying the different mechanisms at play.

\section{Characteristics of Stellar Populations}

We used the integral-field unit OASIS at the Canada-France-Hawai Telescope and William Herschel Telescope, and SpIOMM, the imaging Fourier transform spectrometer of the Observatoire du Mont-Mégantic (Drissen et al., these proceedings), to describe the stellar populations of spiral galaxies. The small $\sim 12^{\prime \prime} \times 12^{\prime \prime}$ OASIS field of view was centered on the nucleus of eight barred spirals. It gave about 1000 spectra per galaxy, in the wavelength range around $\mathrm{H} \beta$ (4700 to $5558 \AA$ ) and $\mathrm{H} \alpha(6210$ to $7008 \AA$ ) with an 
instrument resolution of $\sim 2 \AA \mathrm{pix}^{-1}$. SpIOMM large $12^{\prime} \times 12^{\prime}$ field of view allows us to study the emission lines over the entire disk, with more than $10^{6}$ spectra using two filters centered on $\mathrm{H} \beta$ and $\mathrm{H} \alpha$, of many nearby galaxies (see also Rousseau-Nepton et al., these proceedings)

Cantin (2010) developed an iterative technique to separate the flux from different stellar populations in each OASIS spectrum. In summary : a young population $(<15 \mathrm{Myr})$ is characterized by comparing the equivalent width of $\mathrm{H} \beta$ and $\mathrm{H} \alpha$ with starburst models from LavalSB (Dionne \& Robert 2006) for an instantaneous burst with a standard initial mass function (i.e. a slope of 2.35 with cutoff masses of 1 and $100 \mathrm{M}_{\odot}$ ). Absorption lines of $\mathrm{H} \beta, \mathrm{Mg}_{2}$ at $5177 \AA$, and FeI at 5270 and $5335 \AA$ are used to study directly the stellar populations using instantaneous burst models from Gonzalez Delgado et al. (2005). In most cases, the absorption lines describe an older population than the one seen from the emission lines. The iterative process takes into account the flux and line indicators from one population while studying the other one. The nebular continuum is also taken into account using LavalSB. One advantage of this technique is, for example, to isolate the absorption component in $\mathrm{H} \beta$ and therefore get more accurate age and mass estimates. Reliable uncertainties, within the models considered, are obtained using Bayesian statistics. The detailed analysis of NGC 4900 studied with OASIS and this technique has been published by Cantin et al. (2010). Preliminary results for NGC 5430 have been presented by Robert et al. (2010).

\section{OASIS Results}

Morphology - The morphology in the central galaxy region is very different if we look at the continuum flux or the line emissions. While the continuum flux is generally peaking in the center of the image (but sometimes with nearby multiple maxima; e.g. Fig. 1 and 3), the line emissions may show a nuclear spiral/ring, bar or blobs. Patches of dust appear in between the line emission structures (e.g. Fig. 1). All these structures are often oriented in a specific way in relation with the galaxy bar and arms. The map for all the emission lines observed is identical, except for the two known LINERs, NGC 5921 and NGC 7177. For the latest, the $[\mathrm{NII}] \lambda 6584$ line gets stronger than $\mathrm{H} \alpha$ in a specific region which, interestingly, do not coincide with the continuum peak (see Fig. 1).

Activity - The spectrum for each OASIS pixel often indicates a high ratio [NII] $\lambda 6584 / \mathrm{H} \alpha$ and $[\mathrm{SII}] \lambda 6716+6730 / \mathrm{H} \alpha$ typical for the composite/transition regions defined by BPT (Baldwin et al. 1981). These pixels cover a large portion of the images, extending to more than $1 \mathrm{kpc}$ from the continuum peak. Figure 2 shows the emission line ratios and the BPT diagrams for NGC 4385, as an example. For this galaxy on the other hand, the integrated spectrum (over the OASIS field of view) is typical of an HiI region.

Stellar populations and gas characteristics - The final iteration systematically reveals a high average gas metallicity $12+\log (\mathrm{O} / \mathrm{H})$ close to $2-3 \mathrm{Z}_{\odot}$ in the galaxy central region. Slightly larger values may be observed in some young burst regions (e.g. Fig. 3). The stellar metallicity $[\mathrm{Fe} / \mathrm{H}]$ is usually much lower, from 0.2 to $\mathrm{Z}_{\odot}$, sometimes with a hint of an increase value at the location of the continuum peak. The age of the young populations responsible for emission lines varies between 3 and $12 \mathrm{Myr}$, according to the galaxy. For all galaxies we find a broad distribution, from a few $10 \mathrm{Myr}$ to $\sim 10 \mathrm{Gyr}$, in the age of the stellar populations studied from the absorption lines. These older populations are scattered all over the images.

Histograms of the mass of the young and old stellar populations within each pixel reveal the relative importance of the star forming episodes. The histograms for NGC 5430 are shown in Figure 3. The $\mathrm{S} / \mathrm{N}$ ratio in each spectrum for this object exceeds 40 and an 
internal extinction correction (based on the emission line ratio $\mathrm{H} \alpha / \mathrm{H} \beta$ ) was applied for each pixel, which allows reliable mass estimates. The more recent bursts, around $6.5 \mathrm{Myr}$ (located in the nuclear ring), is an important event of star formation, but we can also see that many bursts of comparable strength have occurred in the past; an important phase of star formation took place around 1 Gyr ago in this galaxy. As shown in the histogram, the gas and stellar metallicity was also fluctuating overtime.

\section{SpIOMM Results}

The SpIOMM study of the bright HII regions in NGC 5430 done by Brière (2009) confirms the high $[\mathrm{NII}] / \mathrm{H} \alpha$ ratio of the central regions, but it also reveals a high ratio for most of the regions in the galaxy bar, arms, and disk. Brière (2009) obtains an age for the central HiI regions which is consistent with the OASIS results. For all the regions studied with SpIOMM, she gets a bimodal distribution peaking at 7.5 and $11 \mathrm{Myr}$.

\section{Summary}

With OASIS, the central region of the barred spiral galaxies studied shows: 1) young star forming knots located in a nuclear spiral/ring, bar or blobs; 2) a peculiar dust distribution; 3 ) older (up to $\sim 10 \mathrm{Gyr}$ ) generations of stars uniformly distributed with a metallicity lower than for the gas; and 4) in most cases, high $[\mathrm{NII}] / \mathrm{H} \alpha$ and $[\mathrm{SII}] / \mathrm{H} \alpha$ ratio extending over large spatial regions. We see relations between the distribution of the star forming knots, dust, regions of higher abundances, and the galaxy large scale bar orientation. These relations confirm the importance of the bar for the galaxy evolution. The long sequence of star forming events is also in good agreement with the model proposed for the formation of a pseudobulge in late type spirals. Many aspects remain to be considered, e.g. gas flow in the central regions (using emission line dynamics) in relation with the metallicity time fluctuation observed, the size of the gas reservoir, the history of interaction... Interesting points have also been raised, e.g. high line ratios.

Integral-field units are ideal to study the components of galaxies. They can reveal many details which may not be seen from an individual spectrum that is covering a large portion of a galaxy or from images with a limited number of wavebands. These details are important clues for the understanding of galaxy evolution.

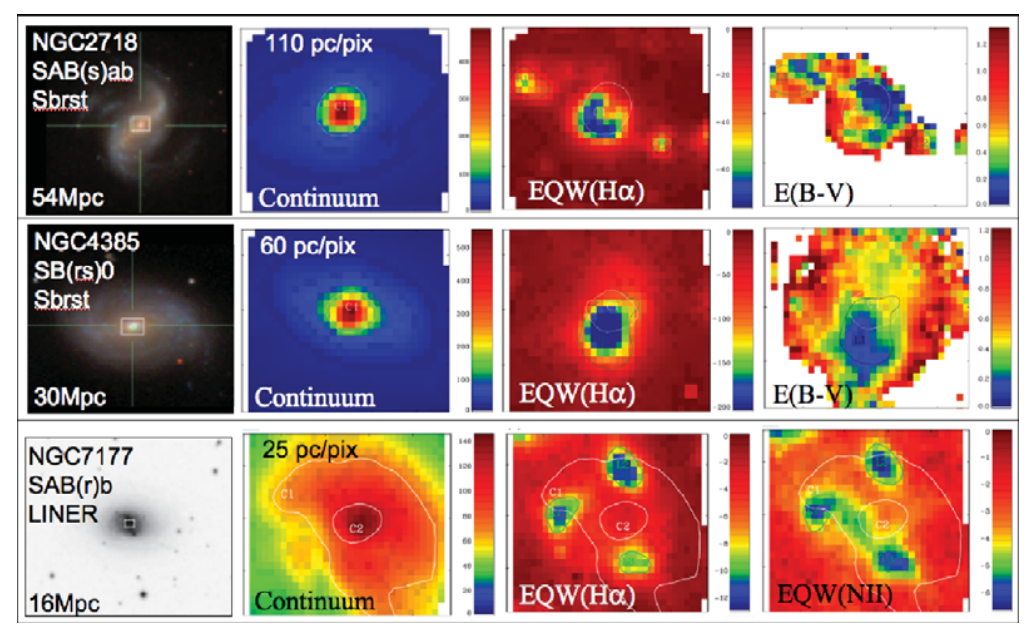

Figure 1. Red continuum, nebular line equivalent width, and E(B-V) maps from OASIS. 


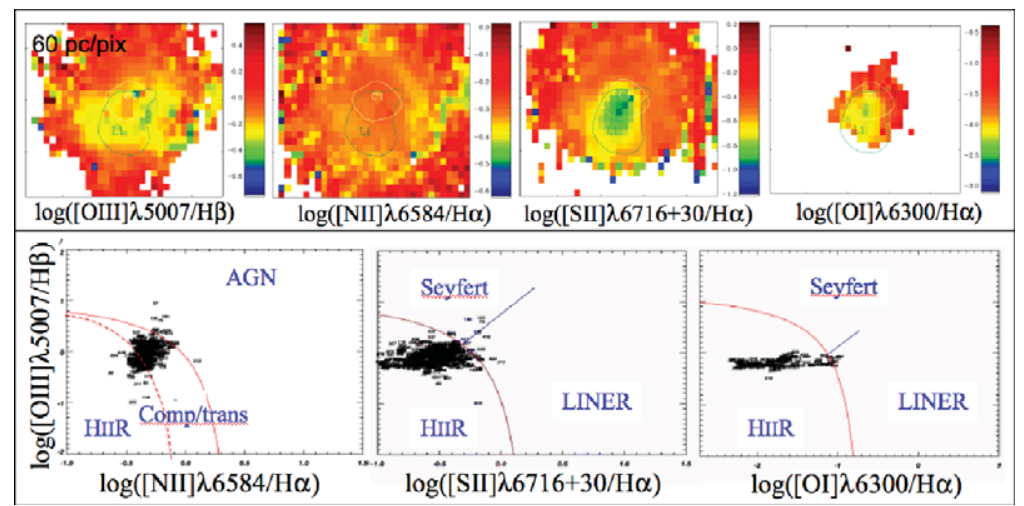

Figure 2. Emission line ratios and BPT diagrams for NGC 4385.

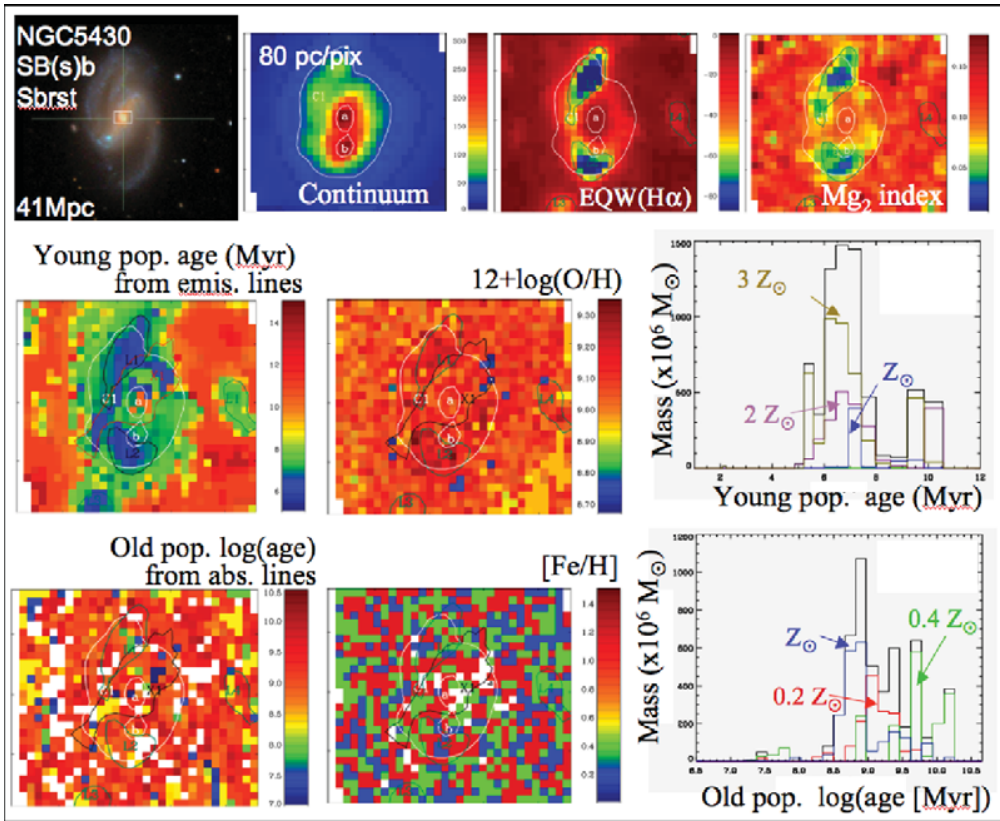

Figure 3. Age, metallcity, and mass of the stellar populations in NGC 5430.

\section{References}

Baldwin, J. A., Phillips, M. M., \& Terlevich, R. 1981, PASP 93, 5

Brière, É. 2009, MSc Thesis Université Laval

Cantin, S., Robert, C., Mollá, M., \& Pellerin, A. 2010, MNRAS 404, 811

Cantin, S. 2010, PhD Thesis Université Laval

Combes, F. 2008, IAU Symp. 245, Formation and Evolution of Galaxy Bulges, 151

Dionne, D. \& Robert, C. 2006, ApJ, 641, 252

Gonzalez Delgado, R. M., Cervino, M., \& Martins, et al. 2005, MNRAS, 357, 945

Haan, S., Schinnerer, E., Emsellem, E., \& Garcia-Burillo, S., et al. 2009, ApJ, 692, 1623

Kormendy, J. \& Kennicutt, Jr.R. C. 2004, ARAA, 42, 603

Norman, C. A., Sellwood, J. A., \& Hasan, H. 1996, ApJ, 462, 114

Ragan, M. W. \& Teuben, P. J. 2004, ApJ, 600, 595

Robert, C., Cantin, S., Molla, M., Pellerin, A., \& Brière, É. 2010, IAU Symp. 262, Stellar populations - Planning for the Next Decade, 418 